MATEC Web of Conferences 25,02007

(2015)

DOI: $10.1051 /$ matecconf/ 20152502007

(C) Owned by the authors, published by EDP Sciences, 2015

\title{
Applied Research of Nanomaterials in Photo-thermal Therapy
}

\author{
Hang Sun \\ Teaching and Research Office of Rehabilitation, Langfang Health Vocational College, Langfang, Hebei, China \\ Xuejun Zuo \\ Teaching and Research Office of Pediatrics, Langfang Health Vocational College, Langfang, Hebei, China
}

Gang Liang

Teaching and Research Office of Computer, Langfang Health Vocational College, Langfang, Hebei, China

\begin{abstract}
In the applied research of nanomaterials in photo-thermal therapy and based on the understanding of the principle of photo-thermal therapy and its medical equipment, this paper analyzes nanomaterials used for photo-thermal therapy, establishes model by the use of comprehensive evaluation method and selects nanomaterials that are suiTable for photo-thermal therapy, namely, carbon nanomaterials and precious metal nanomaterials. In addition, this paper analyzes the importance of human surgical health by the use of photo-thermal therapy and gives considerations from three aspects, that is, the surgical equipment health, the operating room hygiene and the medical health. This paper also establishes a mathematical model through correlation analysis and credibility analysis, thus emphasizing the necessity of surgical health.
\end{abstract}

Keywords: photo-thermal therapy; nanomaterials; human surgery; hygienic problem; mathematical model

\section{INTRODUCTION}

Photo-thermal therapy is one of technologies with a larger development prospect in the current medical community. Its research is not just limited to the theoretical stage of principle analysis. A lot of medical equipment used for photo-thermal therapy have been developed and used clinically.

In the process of using the photo-thermal therapy technology, the key is to use the special photo-thermal therapeutic agents. Such special photo-thermal therapeutic agents are nanomaterials. Zhengbao Cha has researched visualization of the photo-thermal therapy on the basis of analyzing advantages and disadvantages and the limitations of the existing traditional photo-thermal therapeutic agents. He also proposed a new type of photo-thermal therapeutic agent, that is the micro-nano near-infrared absorbent, and he conducted an experimental verification to verify the availability of the micro-nano near-infrared absorbent.

Nanomaterial is not only limited to a single material; nanocomposite also has a certain effect on the photo-thermal therapy, and its effect is more obvious than that of the single nanomaterial. Xinxing Ma has conducted detailed research of the photo-thermal therapy by the use of nanocomposite, in addition, taking graphene oxide-iron oxide nanocomposite as an example and observing the effect of such nanocomposite on the photo-thermal therapy through laboratory experiments.

For photo-thermal therapy, the most important is the cancer treatment. Jian Wang adopts gold nanorods and applies it into the cancer photo-thermal therapy through analysis of its biosensing, so as to open up a new field for the cancer treatment; Naiqiang Yin researches and develops medical nanoprobes, describes the effect of targeted photo-thermal therapy with these nanoprobes, and confirms its feasibility. They all make an outstanding contribution to the development of the medical field.

This paper analyzes nanomaterials used for photo-thermal therapy based on the principle of photo-thermal therapy and its medical equipment, and selects nanomaterials that are suitable for photo-thermal therapy through mathematical modeling, and finally illustrates the importance of human surgical health by the use of photo-thermal therapy, thus laying a foundation for the development and research of technology of photo-thermal therapy.

\section{INTRODUCTION OF PHOTO-THERMAL MODELING}

\subsection{Research background of photo-thermal therapy}

For human beings, tumor is a very terrible disease, regardless of its type, which is very difficult to cure. The emergence of photo-thermal therapy provides a new direction for the tumor treatment. It is a minimally invasive oncotherapy technology, which can significantly reduce the toxicity of the ill system. Therefore, it is considered as one of the most promising technologies for the tumor treatment at present. 


\section{MATEC Web of Conferences}

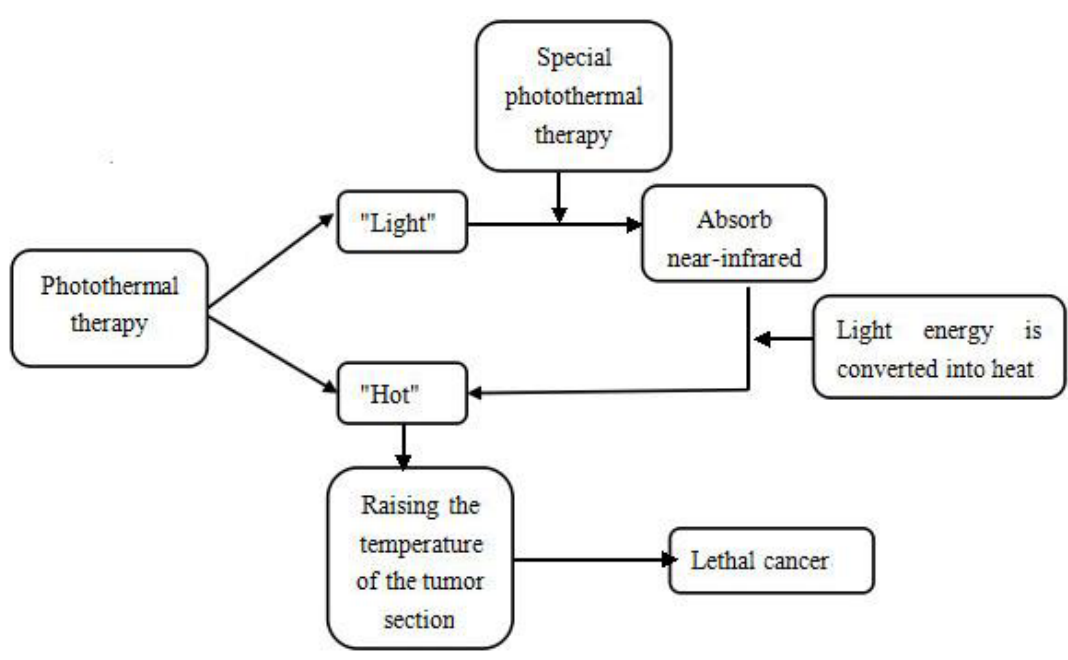

Figure 1. Principle of photo-thermal therapy

\subsection{Principle of photo-thermal therapy and medical equipment}

\subsubsection{Principle of photo-thermal therapy}

For photo-thermal therapy, it implies that "light" and "heat" play important roles. Typically, a special kind of photo-thermal therapeutic agent may be used in photo-thermal therapy, which may absorb near-infrared light under the impact of such photo-thermal therapeutic agent, and improve temperature at the part of tumor by converting light into heat, that is, the part of "heat", and finally reach an effect of killing cancer cells.

The effect of photo-thermal therapy is better than that of traditional tumor treatment. It is an important direction for the medical field to research and develop at the current stage.

\subsubsection{Medical equipment used for photo-thermal therapy}

At the present stage, the technology of photo-thermal therapy has been widely accepted by experts and applied to the treatment of diseases, especially the tumor treatment through clinical studies. Currently, the medical equipment used for photo-thermal therapy is mainly MS-F-1 series of photo-thermal therapeutic equipment, including the dual-cold type, the triple-head type of hot and cold physiotherapy and therapy, different models of photo-thermal therapeutic equipment, and the PTF photo-thermal therapeutic equipment

Wherein PTF photo-thermal therapeutic equipment may enhance flexibility of subcutaneous connective tissue, promote collagen synthesis, improve status quo of skin, and also effectively get rid of the hair, and improve the skin pigmentation and telangiectasia by the use of photo-thermal technology of smooth restoration (PTF) through photopheresis. The photo-thermal technology of smooth restoration adopts high-selectivity light absorption principle with safe operation, light and easy-to-carry instrument. The principle is to use the biostimulation principle and the light pyrolysis principle.

Biostimulation principle: Smooth restoration light can stimulate fibroblasts to produce new collagen and elastic fibers. Rearrangement of collagen and elastic fibers can make the skin become plump, smooth and tight, and restore elasticity. In addition, photo-thermal effect can enhance vascular function, and improve microcirculation, so as to improve wrinkles and shrinkage pores.

Light pyrolysis principle: Since the content of pigment in the lesion tissue is much more than that of normal skin tissue, the temperature produced after absorbing light is also higher than that of the normal skin. The concentricity and selectivity of the light energy act on the pigment, temperature rising and producing photo-thermal effect, crushing pigment, and discharging outside the body through the lymphatic tissue without damage to the normal tissue.

The medical equipment used for photo-thermal therapy has become a sophisticated therapeutic instrument through clinical usage. On the whole, it selectively kills lesion tissue by the use of photo-thermal compound effect to promote metastasis and absorption of inflammation, and ultimately achieve the effect of treatment and complete restoration.

\subsection{Nanomaterials and photo-thermal therapy}

Nanotechnology with a very wide range of application fields is one of the most advanced technologies in today's society. Nanodrugs and nanodrug carriers are most widely used in the medical field. In the medical filed, a variety of nanodrug carriers have been developed. Nano and medicine are combined together by the cutting-edge, multidiscipline use of intersectionality through smallness of nanomaterials. 


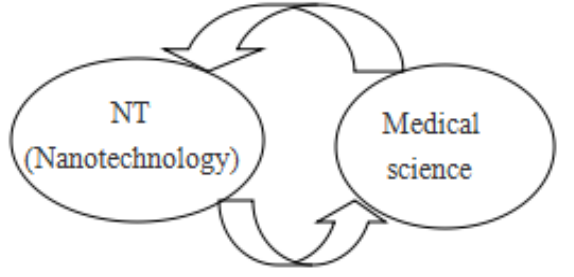

Figure 2. Nanotechnology and medicine

Nowadays, many diseases that are difficult to be cured in medical field can be resolved by nanodrugs. Diabetes, coronary heart disease, hypertension, tumor and cancer can be cured with a very good effect by nanodrugs and nanotechnology. Therefore, research of the application of nanotechnology in the medical field is helpful for its further development and promotion of medical cause.

For nanomaterials used for photo-thermal therapy, there are generally traditional organic compounds, carbon nanomaterials and precious metal nanomaterials. Traditional organic compounds include polyaniline and indocyanine green; carbon nanomaterials include carbon nanotubes and reducing graphene; precious metal nanomaterials include gold bars, gold nanoshells and gold cage.

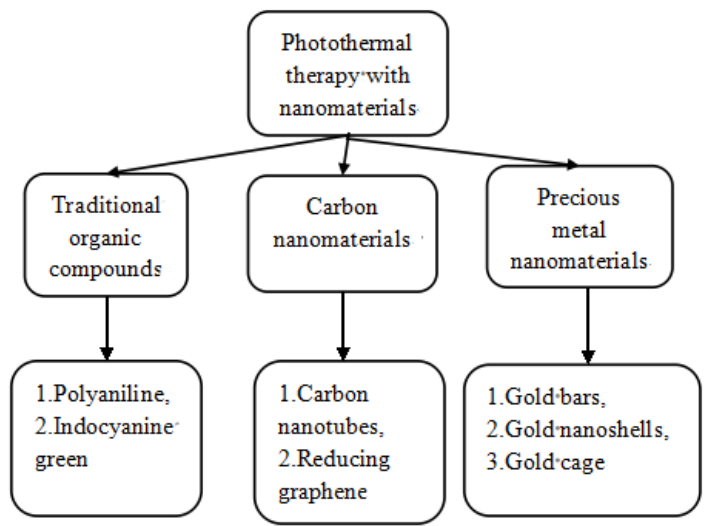

Figure 3. Photo-thermal therapy with nanomaterials

However, traditional organic compounds, carbon nanomaterials and precious metal nanomaterials have some flaws. The biggest problem of traditional organic compounds is that the photo-thermal conversion rate is low, which is harmful for photo-thermal therapy. Carbon nanomaterials have certain toxicity, and the stability and measurement selection are critical. What's worse, the cycle time is not very stable. Thus these we list above are considerations before use. The main problem of precious metal nanomaterials is non-degradability of the gold, which has a significant impact on living organisms, and the other problem is the difficulty in metastasis and ex- cretion. Therefore, to widely use nanomaterials in photo-thermal therapy is a great challenge in clinical research.

\subsubsection{Correlation analysis of photo-thermal therapy and nanomaterials}

In order to analyze the correlation between photo-thermal therapy and nanomaterials, there is a need to conduct quantitative analysis through correlation analysis, and compare its correlation by the size of correlation coefficient.

Correlation analysis is to compare correlation through comparing the size of correlation coefficient by using software processing data. Common correlation coefficients are Pearson correlation coefficient and Spearman rank correlation coefficient. In this paper, the correlation is compared by the use of Pearson correlation coefficient.

The Pearson correlation coefficient is mathematical statistics used for representing the level of similarity between two variables, which can be used to quantitatively calculate the similarity between two variables. The calculation formula is as follows:

$\rho(X, Y)=\frac{\operatorname{cov}(X, Y)}{\sigma_{x} \sigma_{y}}=\frac{E\left(\left(X-\mu_{x}\right)\left(Y-\mu_{y}\right)\right)}{\sigma_{x} \sigma_{y}}$

Where the covariance is a numerator and product of standard deviation of two variables and the standard deviation of two variables should not be 0 .

$\mu_{x}=E(X), \sigma_{X}{ }^{2}=E\left(X-\mu_{x}\right)^{2}=E\left(X^{2}\right)-E^{2}(X)$

So the Pearson correlation coefficient can be written as follows:

$\rho(X, Y)=\frac{E(X Y)-E(X) E(Y)}{\sqrt{E\left(X^{2}\right)-E^{2}(X)} \sqrt{E\left(Y^{2}\right)-E^{2}(Y)}}$

When the Pearson correlation coefficient of two variables is close to 1 or -1 , it indicates that their correlation is large or closely related. When the Pearson correlation coefficient of two variables is close to 1 , it indicates that the positive correlation exists between them; when the Pearson correlation coefficient of two variables is close to -1 , the negative correlation exists between them.

In research of nanomaterials used for photo-thermal therapy, the timeliness, service safety, structure stability and low cost are key factors to limit nanomaterials to be used in photo-thermal therapy. The timeliness, service safety, structure stability and low cost are defined as follows:

Timeliness: the performance of photo-thermal conversion under long-term service condition may not be reduced or be slowly reduced.

Service safety: no toxicity or endangered living body in the process of clinical usage. 
MATEC Web of Conferences

Table 1. Data dependency

\begin{tabular}{|l|l|l|l|l|}
\hline Control variable & Timeliness & Service safety & Structure stability & Low cost \\
\hline Pearson correlation & .894 & .885 & .855 & .802 \\
\hline Significance (bilateral) & .856 & .840 & .825 & .811 \\
\hline
\end{tabular}

Structure stability: After a long period or a certain period of use, its structure will not change.

Low cost: The selection of nanomaterials must be based on the principle of low cost; otherwise it is difficult to achieve a large-scale production.

Therefore, there is a need to establish a model of correlation between photo-thermal therapy and nanomaterials and obtain the following results by using SPSS software for processing.

The Table 1 shows that the correlation coefficient is between four factors-timeliness, service safety, structure stability and low cost. Photo-thermal therapy is above 0.8 , indicating that based on these types of evaluation factors, the photo-thermal therapy is closely related to nanomaterials.

2.3.2 Selected model of nanomaterials used for photo-thermal therapy based on comprehensive assessment system

To further analyze the advantages of three kinds of nanomaterials (traditional organic compounds, carbon nanomaterials and precious metal nanomaterials) used for photo-thermal therapy, this paper selects nanomaterials that are the most suitable for photo-thermal therapy. Now, considering from four aspects of nanomaterials-timeliness, service safety, structure stability and low cost, this paper establishes a selected model of nanomaterials used for photo-thermal therapy based on comprehensive assessment system, thereby determining nanomaterials that are the most favorable for photo-thermal therapy.

First, based on:

$U=\left\{u_{1}, u_{2}, \cdots, u_{m}\right\}, m=1,2,3$

Thereby determine evaluation index set $=\{$ traditional organic compounds, carbon nanomaterials, precious metal nanomaterials $\}$.

To calculate weight by using normalization method, the result is as follows:

$w=\{0.243, \quad 0.303, \quad 0.354\}$

Where the main expressive method of the weight is as follows:

$w=\left\{\mu_{1}, \mu_{2}, \cdots, \mu_{m}\right\}, m=1,2, \cdots 3\left(\sum_{m=1}^{6} \mu_{m}=1\right)$

Determine the fuzzy relation matrix $R$ by using subordinating function method, where:
$R=\left(R_{1}, \quad R_{2}, \quad R_{3}\right)^{T}$

(1) The evaluation scale of Level 1:

$\mu_{i 1\left(u_{i}\right)}=\left\{\begin{array}{cc}0 & u_{i} \geq v_{i 2} \\ -\frac{u_{i}-v_{i 2}}{v_{i 2}-v_{i 1}} & v_{i 1}<u_{i}<v_{i 2} \\ 1 & u_{i} \leq v_{i 1}\end{array}\right.$

(2) The evaluation scale of Level $j$ :

$\mu_{i j\left(u_{i}\right)}=\left\{\begin{array}{cc}0 & u_{i} \leq v_{i j-1} \text { or } u_{i} \geq v_{i j+1} \\ \frac{u_{i}-v_{i j-1}}{v_{i j}-v_{i j-1}} & v_{i j-1}<u_{i}<v_{i j} \\ -\frac{u_{i}-v_{i j+1}}{v_{i j+1}-v_{i j}} & v_{i j} \leq u_{i}<v_{i j+1}\end{array}\right.$

(3) The evaluation scale of Level $n$ :

$\mu_{i n\left(u_{i}\right)}=\left\{\begin{array}{cc}0 & u_{i} \leq v_{i n-1} \\ \frac{u_{i}-v_{i n-1}}{v_{i n}-v_{i n-1}} & v_{i n-1}<u_{i}<v_{i n} \\ 1 & u_{i} \geq v_{i n}\end{array}\right.$

Substitute data into the standard membership function formula with above parameters, and determine membership of all evaluation parameters for each rating level, thus constitute the fuzzy relation matrix $R$, namely:

$R=\left(\begin{array}{lll}0.6 & 0.7 & 0.6 \\ 0.3 & 0.2 & 0.25 \\ 0.1 & 0.1 & 0.15\end{array}\right)$

Via:

$S=w \circ R=\left(\mu_{1}, \mu_{2}, \cdots, \mu_{m}\right) \cdot\left(\begin{array}{cccc}r_{11} & r_{12} & \cdots & r_{1 n} \\ r_{21} & r_{22} & \cdots & r_{2 n} \\ \vdots & \vdots & \vdots & \vdots \\ r_{m 1} & r_{m 2} & \cdots & r_{m n}\end{array}\right)$

$=\left(s_{1}, s_{2}, \cdots, s_{n}\right)$ 


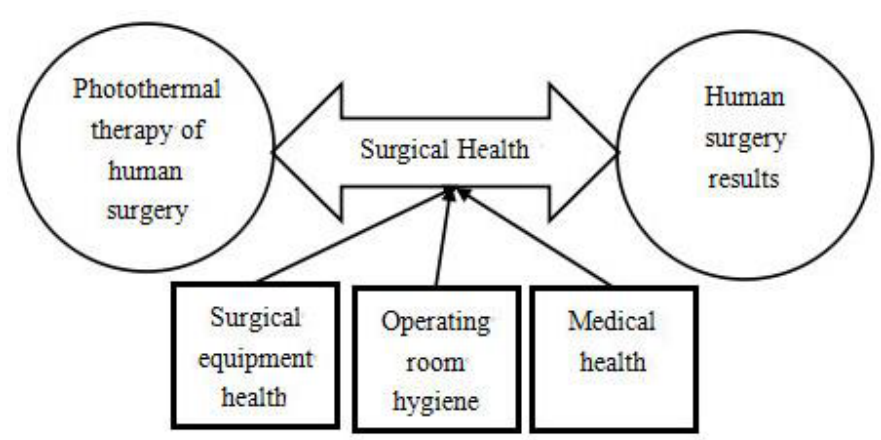

Figure 4. Effectiveness diagram

Table 2. Data dependency

\begin{tabular}{|l|l|l|l|}
\hline Control variable & Surgical equipment health & Operating room hygiene & Medical health \\
\hline Pearson correlation & .894 & .847 & .878 \\
Significance (bilateral) & .805 & .816 & .835 \\
\hline
\end{tabular}

Table 3. Credibility statistics

\begin{tabular}{|l|l|l|l|}
\hline & Cronbach's Alpha & Cronbachs Alpha based on standardized item & Number of item \\
\hline Surgical equipment health & .621 & .975 & 3 \\
Operating room hygiene & .657 & .943 & 3 \\
Medical health & .664 & .952 & 3 \\
\hline
\end{tabular}

Obtain fuzzy evaluation set $S$, where " $\circ$ " is a fuzzy composition operator. Here, taking the fuzzy operator as $M(\cdot, \oplus)$ operator, namely:

$s_{k}=\min \left(1, \sum_{j=1}^{m} \mu_{j} r_{j k}\right), k=1,2, \cdots, n$

Substitute above result into above formula to obtain:

$$
S=\left(\begin{array}{lll}
0.265 & 0.375 & 0.360
\end{array}\right)
$$

Thus, compared with traditional organic compounds, carbon nanomaterials and precious metal nanomaterials have a larger proportion, which are the selected nanomaterials used for photo-thermal therapy.

\subsection{Analysis of photo-thermal therapy and human surgical health based on the mechanism analysis method}

Based on the understanding of the principle of photo-thermal therapy and its medical equipment, this paper analyzes nanomaterials used for photo-thermal therapy, and conducts further research on the selection of nanomaterials. The development of medical equipment and research of nanomaterials that used for photo-thermal therapy lay a foundation for human surgery, thus achieving the purpose of curing human diseases.

When photo-thermal therapy is used for human surgery to eliminate diseases in human bodies such as tumor and so on, hygienic problem is a serious problem that must be considered. Here, this paper will analyze through the mechanism analysis method from three aspects-the surgical equipment health, the operating room hygiene and the medical health.

Everything has its own law of development and basic principles. The mechanism analysis method is set up on this basis, which is a method of scientific research to find and summarize certain development and change rules through analyzing internal rule, reason and mechanism of the things. In the research and analysis of the mechanism of things, the deductive method and mechanism analysis are the same, both of which may be used cooperatively, thus playing a significant role in the research of the mechanism of things.

The mechanism analysis method is often widely-used in physics, chemistry and management circles. Especially in mathematical modeling, the mechanism analysis method is more accurate and closer to reality for analysis of mathematical problems.

For the use of photo-thermal therapy for human surgery, the surgical health is closely related to its effectiveness. The effectiveness diagram is in Figure 4.

In order to analyze the importance of surgical health 


\section{MATEC Web of Conferences}

in the process of using photo-thermal therapy for human surgery, there is a need to compare with their correlation by the use of correlation analysis method. The results are as Table 2.

Thus, it is reasonable to consider the impact of surgical health on the effect of human surgery with photo-thermal therapy from three aspects-surgical equipment health, operating room hygiene and medical health, both of which has a close relationship.

In order to further verify the accuracy of the results, there is a need to further use a new analysis method to evaluate above correlation analysis data and the credibility analysis is to test the credibility of things. In the credibility analysis, the credibility coefficient of Cronbach $\alpha$ is the most commonly-used. Its expression is as follows:

$\alpha=(k /(k-1)) \times\left(1-\left(\sum S_{i}^{2}\right) / S_{T}^{2}\right.$

Compare the size of above coefficient of Cronbach $\alpha$ by using credibility analysis criteria and observe credibility of the system.

Conduct credibility analysis of data in Table 2 by using SPSS software and obtain the following credibility analysis data sheet.

Through above analysis sheet, we can obtain:

Cronbach $\alpha=0.626$

According to the credibility analysis criteria, when

$$
0.5 \leq \text { Cronbach } \quad \alpha<0.7
$$

Credibility is very credible (most common), so the results in Table 2 are reliable. That is, the problem of surgical health is one of important factors to affect human surgery with photo-thermal therapy, and it also emphasizes the importance of hygienic problem.

\section{CONCLUSION}

Based on the understanding of the principle of photo-thermal therapy and its medical equipment, this paper analyzes nanomaterials that are used for photo-thermal therapy, and conducts further research on the selection of nanomaterials. In addition, on this basis, this paper researches hygienic problems in human surgery with photo-thermal therapy.
(1) Considering from four aspects of nanomaterialstimeliness, service safety, structure stability and low cost, the correlation coefficient between the photo-thermal therapies is above 0.8. It indicates that based on these types of evaluation factors, the photo-thermal therapy is closely related to nanomaterials;

(2) Compared with traditional organic compounds, carbon nanomaterials and precious metal nanomaterials have a larger proportion, which are the selected nanomaterials used for photo-thermal therapy;

(3) Considering from three aspects-the surgical equipment health, the operating room hygiene and the medical health, the problem of surgical health is an important factor to affect human surgery with photo-thermal therapy, so there is a need to pay attention to the problem of surgical health.

\section{REFERENCES}

[1] Zhengbao Cha. 2013. Research of micro-nano near-infrared absorbent used for visual photo-thermal therapy. Harbin Institute of Technology.

[2] Naiqiang Yin. 2014. Development of medical nanoprobes and their applications in fluorescent, magnetic labeling and photo-thermal therapy. Southwest University.

[3] Xinxing Ma. 2012. A functionalized graphene oxide iron oxide nanocomposite for magnetically targeted drug delivery, photo-thermal therapy and magnetic resonance imaging. Hubei University.

[4] Jian Wang. 2012. Studies on gold nanorods-based biosensing and cancer photo-thermal therapy. China Ocean University.

[5] Juan Hu. 2014. Development of inorganic nanomaterials and their application in tumor diagnosis and treatment. Anhui University.

[6] Yubin Liu. 2014. Monitoring technology and optimization scheme of tumor photo-thermal therapy based on the photoacoustic spectroscopy. East China Normal University.

[7] Bingluan Liu. 2013. System design and preliminary experimental study of LED photo-thermal therapy based on ARM. Journal of Physical Education.

[8] Xiaoze Shi. 2014. Development of grapheme-based nanocomposite and their applications in tumor imaging and photo-thermal therapy. Hebei Normal University. 